\title{
Comment on "Study of counterintuitive transport properties in the Aubry-André-Harper model via entanglement entropy and persistent current"
}

\author{
Francisco M. Fernández $\odot,{ }^{1, *}$ Diego R. Alcoba $\odot,{ }^{2,3}$ Alicia Torre $\odot,{ }^{4}$ Luis Lain $\odot,{ }^{4}$ Ofelia B. Oña $\odot,{ }^{1}$ and Elias Ríos $\odot{ }^{1}$ \\ ${ }^{1}$ INIFTA, División Química Teórica, Sucursal 4, Casilla de Correo 16, 1900 La Plata, Argentina \\ ${ }^{2}$ Departamento de Física, Facultad de Ciencias Exactas y Naturales, Universidad de Buenos Aires, \\ Ciudad Universitaria, 1428 Buenos Aires, Argentina \\ ${ }^{3}$ Instituto de Física de Buenos Aires, Consejo Nacional de Investigaciones Científicas y Técnicas, \\ Ciudad Universitaria, 1428 Buenos Aires, Argentina \\ ${ }^{4}$ Departamento de Química Física, Facultad de Ciencia y Tecnología, Universidad del País Vasco, \\ Apartado 644, E-48080 Bilbao, Spain
}

(Received 8 January 2020; accepted 30 April 2020; published 19 May 2020)

\begin{abstract}
In a recent study of the transport properties in the Aubry-André-Harper model [Phys. Rev. B 100, 195143 (2019)], the authors point out the failure of the Hellmann-Feynman theorem (HFT) in the case of degenerate states. They try to support such a statement from earlier papers that prove exactly the opposite. It appears that the application of the HFT to degenerate states has not been well understood. For this reason, in this Comment we illustrate the correct application of the HFT to the model just mentioned.
\end{abstract}

DOI: 10.1103/PhysRevB.101.197101

Many years ago, Feynman [1] developed a method for the calculation of forces in molecules that does not require the explicit use of the derivative of the energy. This expression, known as the Hellmann-Feynman theorem (HFT), is discussed in almost every book on quantum mechanics [2] and quantum chemistry [3] and some pedagogical articles discuss its utility in quantum mechanics $[4,5]$. It is worth mentioning its application to perturbation theory [4], even for degenerate states [5].

Some time ago, Zhang and George [6] reported a supposed failure of the theorem in the case of degenerate states and proposed a remedy. The results of such an assessment was curious in light of the fact that the proof of the theorem does not require that the states are nondegenerate [1-5]. Several authors commented on this paper, proving Zhang and George wrong with respect to the failure of the HFT [7-10]. In particular, Fernández [8] showed that the expression for the supposed remedy is correct but unnecessary because the original diagonal HFT is valid for degenerate states provided that one chooses the correct linear combinations of the degenerate eigenfunctions for the calculation. Despite all these further discussions and analysis of the HFT, some researchers still appear to believe that the theorem does not apply to degenerate states [11].

In a recent paper, Roy and Sharma [12] argue that the HFT is not valid for degenerate states and, curiously, look for support from those articles that draw the opposite conclusion [7-10]. In particular, these authors stress the fact that the HFT exhibits discontinuities at the crossings between energy levels. It is worth mentioning that Alon and Cederbaum [7] and Fernández [8] already pointed out that there are no

*fernande@quimica.unlp.edu.ar such discontinuities, but their conclusions seem to have been misinterpreted or gone unnoticed.

In light of the results derived by Roy and Sharma [12], it seems necessary to discuss the HFT for degenerate states in more detail, especially because the model analyzed by those authors exhibits a feature not addressed in those earlier discussions of the theorem [7-10]. In fact, due to level crossings, the lowest energy is a piecewise-defined function of the flux with a discontinuous first derivative [12], which requires a more careful application of the HFT. In this Comment, we derive the HFT, discuss its validity for degenerate states, and apply it to the model discussed by Roy and Sharma [12].

In order to make this Comment sufficiently self-contained, we first summarize the main equations developed in an earlier paper [8]. If the Hamiltonian operator $H(\lambda)$ depends on a parameter $\lambda$, then its eigenvalues $E_{n}$ and eigenfunctions $\psi_{n}$ will also depend on this parameter. For simplicity, we assume that $\left\langle\psi_{m} \mid \psi_{n}\right\rangle=\delta_{m n}$ for all $\lambda$. Under these conditions it can be proved that [8]

$$
\left\langle\psi_{m}\left|\frac{\partial H}{\partial \lambda}\right| \psi_{n}\right\rangle=\frac{\partial E_{n}}{\partial \lambda} \delta_{m n}+\left(E_{n}-E_{m}\right)\left\langle\psi_{m} \mid \frac{\partial \psi_{n}}{\partial \lambda}\right\rangle .
$$

When $m=n$, we obtain the well-known diagonal form of the HFT [1],

$$
\left\langle\psi_{n}\left|\frac{\partial H}{\partial \lambda}\right| \psi_{n}\right\rangle=\frac{\partial E_{n}}{\partial \lambda} .
$$

Notice that the proof of the HFT does not assume that the states are nondegenerate; in fact, in the degenerate case, Eq. (1) becomes

$$
\left\langle\psi_{m}\left|\frac{\partial H}{\partial \lambda}\right| \psi_{n}\right\rangle=0, \quad E_{m}=E_{n}, \quad m \neq n .
$$

Obviously, in the case of degenerate states we have to take into account both Eqs. (2) and (3) simultaneously. 
Suppose that at $\lambda=\lambda_{0}$ the energy level $E_{n}$ is $g_{n}$-fold degenerate,

$$
\begin{aligned}
H \varphi_{n+i} & =E_{n} \varphi_{n+i}, \quad\left\langle\varphi_{n+i} \mid \varphi_{n+j}\right\rangle=\delta_{i j}, \\
i, j & =0,1, \ldots, g_{n}-1 .
\end{aligned}
$$

Clearly, we can construct an infinite number of sets of $g_{n}$ linearly-independent linear combinations of the eigenfunctions $\varphi_{n+i}$,

$$
\psi_{n+i}=\sum_{j=0}^{g_{n}-1} c_{j i} \varphi_{n+j}, \quad i=0,1, \ldots, g_{n}-1,
$$

that are also eigenfunctions of $H$ with eigenvalue $E_{n}$. However, any such set will not necessarily satisfy the HFT unless the coefficients $c_{j i}$ are chosen so that

$$
\left\langle\psi_{n+i}\left|\frac{\partial H}{\partial \lambda}\right| \psi_{n+j}\right\rangle=\frac{\partial E_{n+i}}{\partial \lambda} \delta_{i j}, \quad i, j=0,1, \ldots, g_{n}-1,
$$

in agreement with Eqs. (2) and (3). Notice, for example, that the arbitrary eigenfunctions

$$
\varphi_{n+i}=\sum_{j=0}^{g_{n}-1} c_{i j}^{*} \psi_{n+j}
$$

will not satisfy the diagonal HFT,

$$
\left\langle\varphi_{n+i}\left|\frac{\partial H}{\partial \lambda}\right| \varphi_{n+i}\right\rangle=\sum_{j=0}^{g_{n}-1}\left|c_{i j}\right|^{2} \frac{\partial E_{n+j}}{\partial \lambda},
$$

unless $\partial E_{n+j} / \partial \lambda=\partial E_{n} / \partial \lambda$ for all $j=0,1, \ldots, g_{n}-1$. The latter particular condition takes place, for example, when the variation of $\lambda$ does not change the symmetry of the problem and the degeneracy is not removed. It is clear that the diagonal elements of $\partial H / \partial \lambda$ calculated with arbitrary degenerate eigenfunctions of $H$ at $\lambda=\lambda_{0}$ will simply yield averages of the actual slopes of the eigenvalues. The actual slopes are given by those eigenfunctions that satisfy Eq. (6). It is obvious that this condition can always be satisfied because the coefficients $c_{j i}$ are given by a straightforward diagonalization of the $g_{n} \times g_{n}$ Hermitian matrix representation of $\partial H / \partial \lambda$ at $\lambda=\lambda_{0}$.

Although these arguments were clearly stated in an earlier paper [8], Roy and Sharma [12] have suggested that the HFT breaks down at degeneracies in the energy spectrum and showed results that apparently support this conclusion (see the discrepancy between "fwd. diff." and "FH theo." in their Fig. 13). However, it has been clearly shown that not only is the HFT strictly valid at degeneracies, but that there is no discontinuity whatsoever [7,8]. In fact, the degenerate eigenfunctions that satisfy Eqs. (2) and (3) at $\lambda_{0}$ are given by the continuity equation

$$
\psi_{n}\left(\lambda_{0}\right)=\lim _{\lambda \rightarrow \lambda_{0}} \psi_{n}(\lambda),
$$

and all the mathematical relationships, such as (1), for example, are continuous at $\lambda_{0}$. It can be shown that the discrepancy between the left- and right-hand sides of Eq. (2) found by Roy and Sharma [12] is the result of a wrong choice of the eigenfunctions at the level crossings. It is worth noticing that those authors comment on the diagonalization of $\partial H / \partial \lambda$ at the level crossings but do not resort to it in order to obtain the correct results.

The model chosen by Roy and Sharma [12] is the AubryAndré-Harper (AAH for short) one given by the phasetwisted, one-particle Hamiltonian operator

$$
\begin{aligned}
H(\theta)= & -J \sum_{l=1}^{N}\left(e^{-i \theta / N} c_{l}^{\dagger} c_{l+1}+e^{i \theta / N} c_{l+1}^{\dagger} c_{l}\right) \\
& +\xi \sum_{l=1}^{N} \cos (2 \pi \alpha l) c_{l}^{\dagger} c_{l},
\end{aligned}
$$

where $J$ is the strength of the nearest-neighbor hopping, $c_{l}$ is the fermionic annihilation operator acting on site $l, \theta=$ $2 \pi \phi / \phi_{0}, \phi$ the magnetic flux, $\phi_{0}=\hbar / e$ the unit of flux quanta, and $\alpha$ an irrational number. In order to discuss the validity of the HFT for degenerate states, the authors consider the strength $\xi$ of the quasiperiodic potential equal to zero (onedimensional tight-binding chain) and, therefore, we omit the second term on the right-hand side from now on that may also contain a phase $\theta_{p}$ that the authors neglect in some of their calculations. The Hamiltonian operator (10) describes a lattice on a ring of $N$ sites. The eigenvalue equation for this model can be solved exactly and the single-particle energies are given by

$$
\epsilon_{n}(\phi)=-2 J \cos \left[\frac{2 \pi}{N}\left(n+\frac{\phi}{\phi_{0}}\right)\right], \quad-\frac{N}{2} \leqslant n<\frac{N}{2} .
$$

The authors analyze the persistent current given by

$$
I_{c}=-\frac{\partial E_{0}}{\partial \phi},
$$

where [12]

$$
E_{0}(\phi)=\sum_{n} \epsilon_{n}(\phi) \theta\left(E_{F}-\epsilon_{n}\right)
$$

is the lowest energy of the system of $N_{p}$ spinless Fermions. In this equation, $E_{F}$ is the Fermi energy at zero temperature and $\theta(x)$ the Heaviside step function. Although $E_{0}(\phi)$ is continuous (because the number of single-particle energies in the sum remains constant) it will have a discontinuous first derivative $\partial E_{0} / \partial \phi$ at every crossing point between the two lowest system energies. In such cases the HFT still applies to each piece of a piecewise-defined function and most care should be taken at the joints. This is a particular feature of this application of the AAH model that was not addressed in previous discussions of the HFT [7-10].

In order to make the present discussion as simple as possible, we first consider the matrix representation of a oneparticle, three-site Hamiltonian,

$$
\mathbf{H}=-\left(\begin{array}{lll}
0 & e^{-i \theta / 3} & e^{i \theta / 3} \\
e^{i \theta / 3} & 0 & e^{-i \theta / 3} \\
e^{-i \theta / 3} & e^{i \theta / 3} & 0
\end{array}\right),
$$

where we have chosen $J=1$ and $\xi=0$. Figure 1 shows the one-particle energies for this simple model (which are labeled $j=1,2,3$ instead of $j=-1,0,1)$. For simplicity, we choose $\phi_{0}=1$, so that $\theta=2 \pi \phi$, and in the chosen range of values of the model parameter the one-particle energies undergo crossings at $\phi=-1,-0.5,0,0.5,1$. Figure 2 (upper 


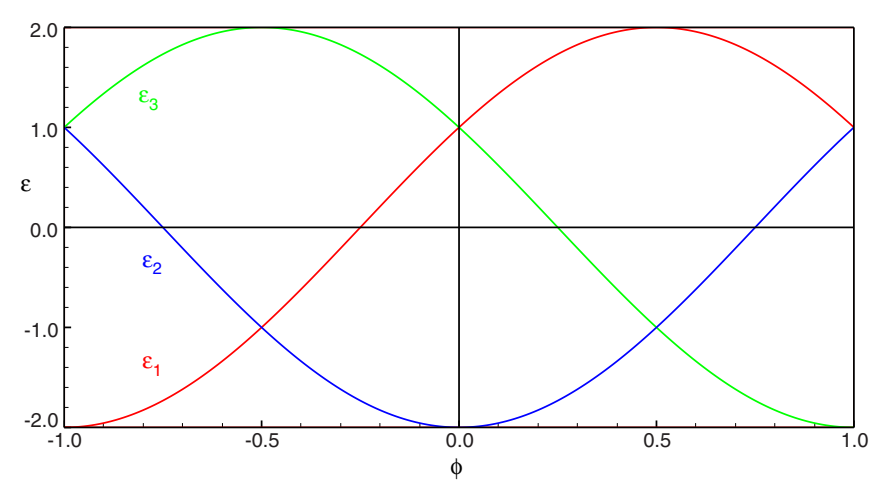

FIG. 1. Single-particle energies of model, Eq. (14).

panel) shows the system energies $E=\epsilon_{j}+\epsilon_{k}, j \neq k$, for two spinless fermions as functions of the model parameter $\phi$ and we appreciate that there are crossings at the $\phi$ values just mentioned. In what follows, we focus on the one at $\phi=0$ where the two lowest-energy levels exchange roles as the ground and first excited ones (notice that the same behavior takes place at $\phi= \pm 1)$. The resulting lowest-energy level $E_{0}(\phi)$ exhibits a cusp at $\phi=0$ due to the crossing just mentioned. Consequently, $d E_{0} / d \phi$ is discontinuous at $\phi=0$ as shown in the lower panel. As argued above, the HFT applies to $\phi<0$ and $\phi>0$ and one obtains the exact result at $\phi=0$ from the limits $\phi \rightarrow 0^{ \pm}$. However, in what follows, we proceed in a naive way and solve the eigenvalue equation for the matrix (14) with $\phi=0$ without using the information of what happens at any other $\phi$ point. Three arbitrary orthonormal
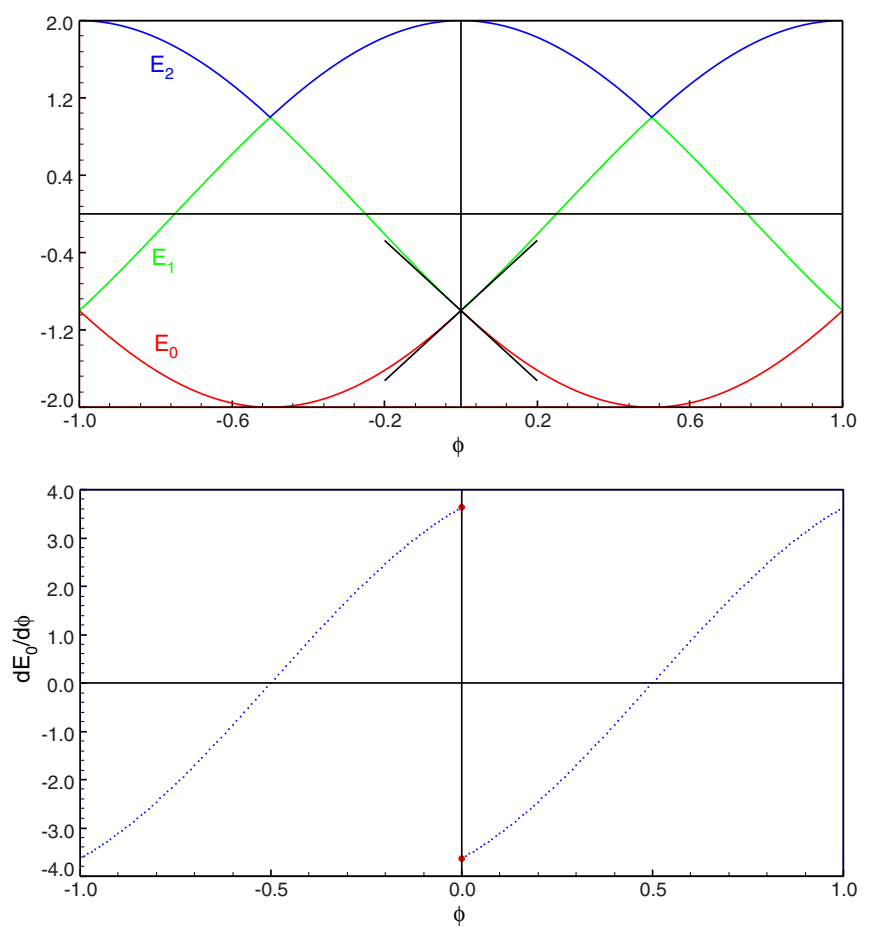

FIG. 2. System energies $E_{k}$ for model, Eq. (14), and first derivative $d E_{0} / d \phi$.
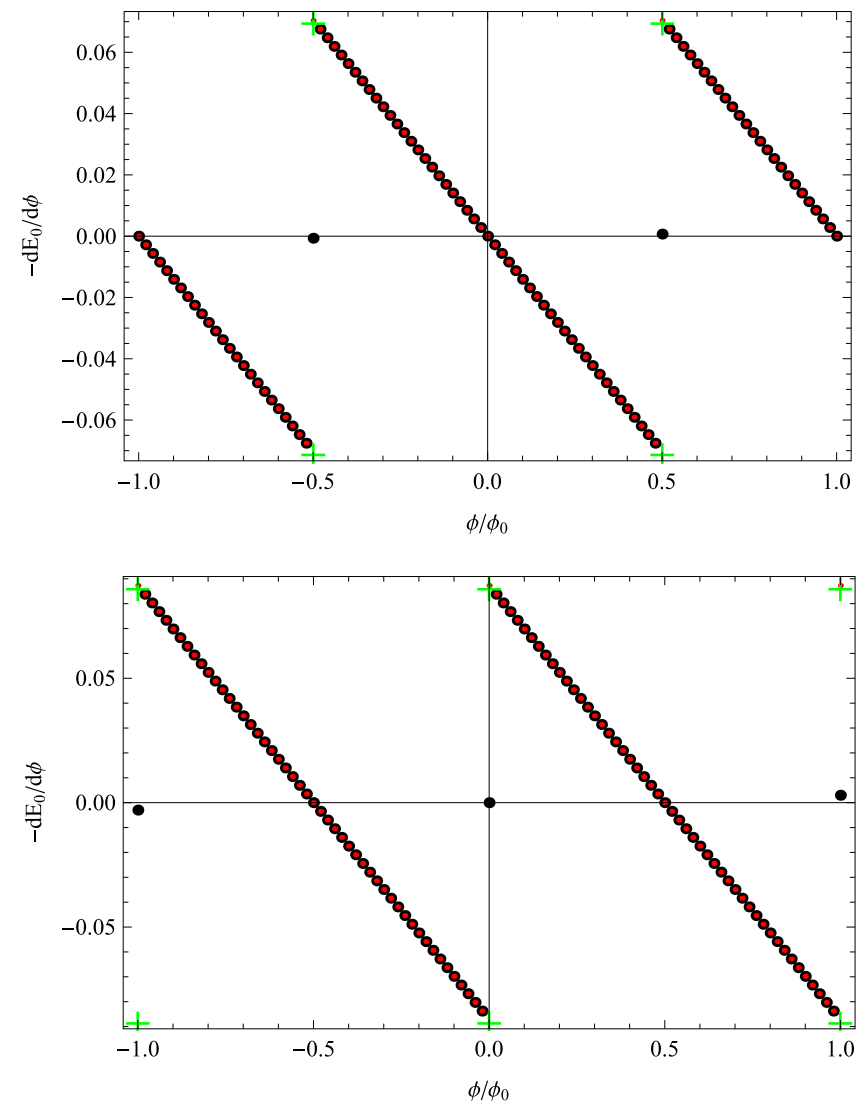

FIG. 3. First derivative $-d E_{0} / d \phi$ for the one-dimensional tightbinding chain $[J=1, \xi=0$ in Eq. (10)] with $N=144, v=0.3$ (upper panel), and $v=0.5$ (lower panel).

eigenvectors are

$$
\mathbf{v}_{1}=\frac{1}{\sqrt{3}}\left(\begin{array}{l}
1 \\
1 \\
1
\end{array}\right), \quad \mathbf{v}_{2}=\frac{1}{\sqrt{2}}\left(\begin{array}{r}
1 \\
0 \\
-1
\end{array}\right), \quad \mathbf{v}_{3}=\frac{1}{\sqrt{6}}\left(\begin{array}{r}
-1 \\
2 \\
-1
\end{array}\right)
$$

corresponding to single-particle energies $\epsilon_{1}=-2, \epsilon_{2}=\epsilon_{3}=1$. The $2 \times 2$ matrix representation of $d \mathbf{H} / d \phi$ at $\phi=0$ in the subspace spanned by $\mathbf{v}_{2}$ and $\mathbf{v}_{3}$ is

$$
\left.\frac{d \mathbf{H}}{d \phi}\right|_{2 \times 2}(\phi=0)=\frac{2 \pi i}{\sqrt{3}}\left(\begin{array}{rr}
0 & 1 \\
-1 & 0
\end{array}\right),
$$

with eigenvalues $\pm 2 \pi / \sqrt{3}$. Since $d \epsilon_{1} /\left.d \phi\right|_{\phi=0}=0$, then the two slopes of $d E_{0} /\left.d \phi\right|_{\phi=0}$ are given by these two eigenvalues. They are marked by straight lines and red circles in the upper and lower panels, respectively, of Fig. 2. We stress that the diagonal elements of the matrix (16) give wrong slopes at $\phi=0$ because the eigenvectors $\mathbf{v}_{2}$ and $\mathbf{v}_{3}$ do not satisfy Eq. (6). These wrong slopes are equivalent to the out-of-place triangles in Fig. 13 of Roy and Sharma [12]. The straight lines through $\phi=0$ in the upper panel of Fig. 2 clearly illustrate the concept of continuity of the equations of the HFT pointed out by Eq. (9).

Figure 3 shows $-d E_{0} / d \phi$ for the AAH model (10) with $J=1, \xi=0, N=144$, and filling fractions $v=0.3$ (upper panel) and $v=0.5$ (lower panel) $\left(v=N_{p} / N\right)$ calculated 
by means of the right-hand side (red points) and left-hand side (black points) of the diagonal HFT, Eq. (2). In the latter case we obtained the slope by means of a straightforward expectation value using an arbitrary eigenfunction at the discontinuities (black points) and also by means of the proper eigenfunctions obtained from diagonalization of $d \mathbf{H} / d \phi$ (green crosses). This figure is similar to Fig. 13 of Roy and Sharma [12] and shows that the HFT always gives the correct slope (green crosses) if one proceeds carefully at the crossing points. The out-of-place triangles in Fig. 13 of Roy and Sharma (as well as some black points in the present Fig. 3) are simply due to a wrong application of the HFT.

Throughout this Comment we have tried to make it clear that the diagonal HFT, Eq. (2), is valid in the case of degenerate states, as argued in several papers [7-10]. Any discrepancy between the two ways of calculating the slopes of the energy levels [left- and right-hand sides of Eq. (2)] arises from the wrong choice of the eigenfunctions used in the calculation of the expectation values at a level crossing. The correct degenerate eigenfunctions are those that satisfy Eq. (6). This condition is not a correction of the HFT as misinterpreted by Roy and Sharma [12] because the functions that satisfy it are given naturally by the continuity Eq. (9). There is no discontinuity whatsoever at a level crossing as clearly follows from the continuity equation just mentioned. However, the definition of the ground-state energy as in Eq. (13) forces a discontinuity in $\partial E_{0} / \partial \phi$ because the energy $E_{0}$ is given by one state on one side of a level crossing and a different state on the other side of it (piecewise-defined function). At the cusp generated by the level crossing, one has to choose the correct eigenfunctions that are given by Eq. (6). The simple example discussed above clearly illustrates these points.

We want to stress the fact that at a crossing of $g_{n}$ energy levels, one expects $g_{n}$ slopes. If one does not have information about what happens in the neighborhood of the crossing, one should carry out the calculation according to Eq. (6) that yields the slope with which every energy level approaches or leaves the crossing. The use of arbitrary eigenfunctions leads to the wrong slopes shown by Eq. (8). If, on the other hand, one manages to follow a given energy level through the crossing, one should not have any difficulty in obtaining its slope by means of the left- and right-hand sides of Eq. (2).

In general, the states that cross at some value of the model parameter have different symmetries (see Ref. [13] and references therein). These states obviously satisfy Eq. (6). Consequently, if one carries out calculations for each symmetry species separately [14], no crossing occurs and one is not forced to construct the degenerate eigenfunctions that satisfy the HFT (the theorem is automatically satisfied for each irreducible representation). Any arbitrary linear combination of degenerate eigenfunctions mixes different symmetries and one obtains the wrong result shown in Eq. (8). An example is given by the triangles in Fig. 13 of Roy and Sharma [12]. These conclusions are also valid for approximate variational wave functions. Of course this analysis should be carefully applied to the case in which one is forced (for physical reasons) to choose always the lowest-energy level $E_{0}$ because it is related to one irreducible representation when $\lambda<\lambda_{0}$ and another one for $\lambda>\lambda_{0}$. This pathological situation takes place in the study of the transport properties in the AAH model discussed by Roy and Sharma [12].

Finally, we want to point out that the present discussion is restricted to $T=0$. The form of the HFT for statistical averages $(T>0)$ has already been discussed [11,15], but the continuity for degenerate states still requires further analysis [11].

We expect that the present Comment will make the issue of the HFT for degenerate states clearer than the previous one [8].

The authors acknowledge financial support from the Universidad de Buenos Aires (Grant No. 20020150100157BA), the Consejo Nacional de Investigaciones Científicas y Técnicas (Grant No. PIP 11220130100377CO, No. PIP $11220130100311 \mathrm{CO}$, and No. 2013-1401PCB), and the Agencia Nacional de Promoción Científica y Tecnológica (Grant No. PICT-201-0381), Argentina. E.R. acknowledges support to Consejo Nacional de Investigaciones Científicas y Técnicas.
[1] R. P. Feynman, Phys. Rev. 56, 340 (1939).

[2] C. Cohen-Tannoudji, B. Diu, and F. Laloë, Quantum Mechanics (Wiley, New York, 1977).

[3] F. L. Pilar, Elementary Quantum Chemistry (McGraw-Hill, New York, 1968).

[4] S. T. Epstein, Am. J. Phys. 22, 613 (1954).

[5] S. B. Singh and C. A. Singh, Am. J. Phys. 57, 894 (1989).

[6] G. P. Zhang and T. F. George, Phys. Rev. B 66, 033110 (2002).

[7] O. E. Alon and L. S. Cederbaum, Phys. Rev. B 68, 033105 (2003).
[8] F. M. Fernández, Phys. Rev. B 69, 037101 (2004).

[9] S. R. Vatsya, Phys. Rev. B 69, 037102 (2004).

[10] R. Balawender, A. Holas, and N. H. March, Phys. Rev. B 69. 037103 (2004).

[11] D. Rai, Phys. Rev. A 75, 032514 (2007).

[12] N. Roy and A. Sharma, Phys. Rev. B 100, 195143 (2019).

[13] F. M. Fernández, J. Math. Chem. 52, 2322 (2014).

[14] P. Amore and F. M. Fernández, arXiv:1911.04909

[15] F. M. Fernández, J. Math. Chem. 52, 2128 (2014). 